\title{
The glypican 3 oncofetal protein is a promising diagnostic marker for hepatocellular carcinoma
}

\author{
Naoko Yamauchi ${ }^{1}$, Akira Watanabe ${ }^{2}$, Michiyo Hishinuma ${ }^{1}$, Ken-ichi Ohashi ${ }^{1}$, \\ Yutaka Midorikawa ${ }^{3}$, Yasuyuki Morishita ${ }^{1}$, Toshiro Niki ${ }^{1}$, Junji Shibahara ${ }^{1}$, \\ Masaya Mori ${ }^{4}$, Masatoshi Makuuchi ${ }^{3}$, Yoshitaka Hippo ${ }^{2}$, Tatsuhiko Kodama ${ }^{2}$, \\ Hiroko Iwanari $^{5}$, Hiroyuki Aburatani ${ }^{2}$ and Masashi Fukayama ${ }^{1}$ \\ ${ }^{1}$ Department of Pathology, Graduate School of Medicine, University of Tokyo, Tokyo, Japan; ${ }^{2}$ Genome Science \\ Division, Research Center for Advanced Science and Technology, University of Tokyo, Tokyo, Japan; \\ ${ }^{3}$ Hepato-Biliary-Pancreatic Surgery, Graduate School of Medicine, University of Tokyo, Tokyo, Japan; \\ ${ }^{4}$ Mitsui Memorial Hospital, Tokyo, Japan and ${ }^{5}$ Perseus Proteomics, Inc., Tokyo, Japan
}

\begin{abstract}
Expression profiling of hepatocellular carcinoma has demonstrated that glypican 3 (GPC3), a heparan sulfate proteoglycan anchored to the membrane, is expressed at a markedly elevated level in hepatocellular carcinoma. In this paper, two monoclonal antibodies against GPC3, GPC3-C02 and A1836A, were confirmed to specifically recognize GPC3molecule in cells from hepatocellular carcinoma and hepatoblastoma cell lines by immunoblotting, and both were confirmed to recognize different epitopes of the GPC3 molecule by epitope mapping. Then, we evaluated the feasibility of GPC3-immunohistochemistry in the pathological diagnosis of benign and malignant hepatocellular lesions by applying these monoclonal antibodies to formalin-fixed and paraffin-embedded specimens. The immunoreactivity turned out to be identical in the two monoclonal antibodies and was thus confirmed to represent the actual expression of the GPC3 molecule. The expression was observed in the fetal liver, but not in normal adult liver, liver cirrhosis or hepatitis except for a tiny focus of a regenerative nodule of fulminant hepatitis. Diffusely positive staining of GPC3 was observed in malignant hepatocytes in hepatoblastomas and in hepatocellular carcinomas (47/56, 84\%). GPC3 expression was independent of the differentiation and size of the hepatocellular carcinoma. On the other hand, there was only weak and focal staining in low-grade (2/8) and high-grade dysplastic nodules (6/8). GPC3 immunoreactivity was detected in only one of $\mathbf{2 3}$ metastatic lesions of colorectal carcinoma, and its expression was entirely absent in the liver cell adenoma (0/7), carcinoid tumor (0/1), and cholangiocellular carcinoma (0/16). When compared with immunohistochemistry of hepatocyte antigen and alpha-fetoprotein, GPC3-immunohistochemistry was siginificantly much more specific and sensitive for hepatocellular carcinomas. Thus, GPC3 was confirmed to be one of the oncofetal proteins now attracting attention for their promise both as markers of hepatocellular carcinoma in routine histological examination and as targets in monoclonal antibody-based hepatocellular carcinoma therapy.
\end{abstract}

Modern Pathology (2005) 18, 1591-1598. doi:10.1038/modpathol.3800436; published online 13 May 2005

Keywords: glypican 3; hepatocellular carcinoma; immunohistochemistry

Hepatocellular carcinoma is already one of the world's most common malignancies, and its incidence is rapidly increasing in both Asian and Western countries. ${ }^{1,2}$ With the advancement of imaging analyses techniques such as ultrasound

Correspondence: Dr N Yamauchi, MD, PhD, Department of Pathology, Graduate School of Medicine, University of Tokyo, 7-3-1, Hongo, Bunkyo-ku, Tokyo 113-0033, Tokyo, Japan.

E-mail: yamauchi-tky@umin.ac.jp

Received 9 November 2004; revised and accepted 28 March 2005; published online 13 May 2005 imaging, contrast enhanced helical computed tomography, and dynamic magnetic resonance imaging, more and more oncologists are detecting hepatic nodular lesions and having them biopsied for the pathological diagnosis of hepatocellular carcinoma. The distinctions among different hepatocellular nodular lesions are often elusive and challenging for pathologists, however. While reticulin staining and (more recently) CD34-immunohistochemistry have been recognized as helpful diagnostic markers in differential diagnoses among hepatic various lesions (hepatocellular carcinoma, 
dysplastic nodules, and other benign hepatocellular lesions), their usefulness is still somewhat limited. ${ }^{3,4}$ Another difficult challenge in the diagnosis of hepatocellular carcinoma is the differentiation from primary hepatic cholangiocellular carcinoma and liver metastases. Differentiation markers for immunohistochemistry have included hepatocyte antigen (Hep), alpha-fetoprotein (AFP), cytokeratin protein profiling, and the presence of intercellular canaliculi demonstrated by the monoclonal antibodies carcinoembryonic antigen Gold 5 and B-lymphocyte marker CD10. As the variability of these markers might suggest, however, their specificity and sensitivity are somewhat limited for the diagnosis of hepatocellular carcinoma. ${ }^{5}$

Glypican 3 (GPC3) is one of the cell surface heparan sulfate proteoglycans that bind to the cell membrane via glycosilphosphatidylinositol anchors. ${ }^{6}$ Heparan sulfate proteoglycans are well known to interact with growth factors through heparan sulfate chains and thereby to serve as coreceptors for heparin-binding growth factors. ${ }^{7-9}$ While studies on ovarian cancer cell lines, mesotheliomas, and breast tumors have demonstrated the downregulation of GPC3, ${ }^{10-12}$ other investigations on hepatocellular carcinoma have shown a marked elevation of GPC3 mRNA over the level observed in corresponding normal tissues. ${ }^{13-16}$ In the present study, we report the generation of GPC3-C02 and A1836A, a pair of anti-human GPC3 mouse monoclonal antibodies that can detect GPC3 in paraffinembedded tissues by an immunohistochemical technique. When these monoclonal antibodies against GPC3 were applied to malignant and nonmalignant liver tissue samples, we confirmed that they had high sensitivity and specificity not only as diagnostic markers, but as differentiation markers as well. Our studies show that antibody directed against the oncofetal protein GPC3 has great promise as an immunohistochemical marker of hepatocellular carcinoma. These observations also suggest the possibility of developing a monoclonal antibody for hepatocellular carcinoma therapy.

\section{Materials and methods}

\section{Tissue Samples}

Liver tissue samples were chosen from the pathology files of the Department of Pathology, University Hospital and Graduate School of Medicine, University of Tokyo. A total of 56 hepatocellular carcinomas consisting of 18 well differentiated, 29 moderately differentiated, and nine poorly differentiated tumors were available. The diseases in the 56 noncancerous liver tissues (HBV $n=20$, HCV $n=36$ ) adjacent to the hepatocellular carcinomas were liver cirrhosis in 43 cases and chronic viral hepatitis in 13 cases. Other non-neoplastic lesions (obtained by total hepatectomy) consisted of fulminant hepatitis $(n=9)$, autoimmune hepatitis $(n=2)$, primary sclerosing cholangitis $(n=1)$, and primary biliary cirrhosis $(n=2)$. Three focal nodular hyperplasia samples were obtained by partial hepatectomy. The benign or borderline hepatic lesions included seven liver cell adenomas and 16 dysplastic nodules (eight low-grade and eight high-grade dysplastic nodules, respectively). Other malignant hepatic lesions included three sarcomatoid hepatocellular carcinomas, six hepatoblastomas, 16 cholangiocellular carcinomas, one carcinoid tumor, and 23 liver metastases of colorectal carcinoma. Normal adult $(n=25)$ and fetal livers $(n=9,18-39$ gestational week) were obtained at autopsy. Diagnosis of hepatocellular lesions was according to criteria set forth by the International Working Party. ${ }^{17}$

All of the specimens were formalin-fixed and embedded in paraffin. Tissue sections sliced to a thickness of $4 \mu \mathrm{m}$ were placed on silanated slides and subjected to immunohistochemical studies.

\section{Cell Lines}

The human hepatocellular carcinoma cell lines (Li7, Huh7, and HLE), hepatoblastoma cell line (Huh6), ${ }^{18}$ and lung cancer cell line (A549) were used in this study.

\section{Generation of Anti-GPC3 Monoclonal Antibodies}

Monoclonal antibodies for GPC3 were generated by the previously described method. ${ }^{19}$ We began by preparing a CHO cell line that stably expressed FLAG-tagged GPC3 protein lacking the carboxyl terminal GPI anchor (GPC3 $\Delta$ GPI). Next, we purified GPC3 $\Delta$ GPI by incubating the protein with antiFLAG M2 antibody beads (Sigma, St Louis, MO, USA) and FLAG peptide (Sigma) and then gel-filtrating the product with HiLoad 26/60 Superdex200pg (Amersham Bioscience). Finally, we concentrated the recombinant protein using DEAE Sepharose FF. Purified immunogen was immunized to BALB/c mice. Hybridomas were selected by enzyme-linked immunosorbent assay (ELISA) against the recombinant GPC3 protein, followed by cloning with limited dilution. Glutathione $S$-transferase-fused GPC3 was used to epitope-map the two clones used in this study (A1836A and GPC3-C02) to the amino- and carboxyl-terminal portions of GPC3, respectively.

\section{Immunoblotting}

Total cell lysates were obtained after lysis in $10 \mathrm{mM}$ Tris, pH 7.4, $150 \mathrm{mM} \mathrm{NaCl,} 5 \mathrm{mM}$ EDTA, 1.0\% Triton $\mathrm{X}-100,1.0 \%$ sodium deoxycholate, $0.1 \%$ SDS with protease inhibitor cocktail (Sigma, St Louis, MO, USA). Immunoblotting with cell lines was performed as previously described. ${ }^{20}$ The proteins were separated with $15 \%$ SDS-PAGE, transferred to PVDF 
membrane Hybond $\mathrm{P}$ (Amersham Biosciences), treated with $2 \%$ nonfat milk in TBS containing $0.05 \%$ Tween-20 (TBST), and incubated separately with anti-GPC3 mAb in TBST followed by secondary antibody conjugated to horseradish peroxidase (Amersham Biosciences) at a dilution of 1:5000 diluted in TBST. The protein was visualized using the ECL Plus detection system (Amersham Biosciences).

\section{Immunohistochemistry}

The tissue sections on silanated slides were deparaffinized with xylene, rehydrated with a series of graded alcohols, processed with an autoclave in $10 \mathrm{mmol} / \mathrm{l}$ citrate buffer, $\mathrm{pH}$ 6, treated with horse serum albumin to block nonspecific staining, and immunostained using anti-GPC3 monoclonal antibodies, GPC3-C02 and A1836A by the avidin-biotin horseradish peroxidase method (Vectastain Elite ABC kit, Vector, Burlingame, CA, USA). A slide omitting the primary antibody was used as a negative control. The incubation with the primary antibody was performed at $4^{\circ} \mathrm{C}$ overnight in a moisture chamber. After the treatment with biotinylated anti-mouse IgG, the slide-mounted sections were immersed in methanol containing $0.3 \%$ hydrogen peroxide to block endogenous peroxidase activity, stained with diaminobenzidine with hydrogen peroxide, and counterstained with hematoxylin. Cell membrane and cytoplasmic immunoreactivity were assessed, using the following criteria. All cases with $\geq 10 \%$ positive cells were regarded as either focally positive $(+)(10-50 \%)$ or diffusely positive $(++)(>50 \%)$.

\section{Comparison with Other Hepatocellular Markers}

To investigate the usefulness of an immunohistochemical marker for hepatocellular carcinoma, parallel sections of 45 cases of hepatocellular carcinoma were further stained with primary antibodies to Hep (1:50, Dako Cytomation, Copenhagen, Denmark) and AFP (1:200, Dako Cytomation).

\section{Statistical Analysis}

Statistical analysis of the results was performed using the $\chi^{2}$ test or Fisher's exact test. Differences were considered to be significant at $P<0.05$.

\section{Results}

\section{Immunoblotting of GPC3 in Human Cancer Cell Lines}

GPC3 was immunoblotted with cancer cell lines, which were derived from hepatocellular carcinoma and hepatoblastoma, to confirm the specificity of both anti-GPC3 antibodies, A1836A and GPC3-C02

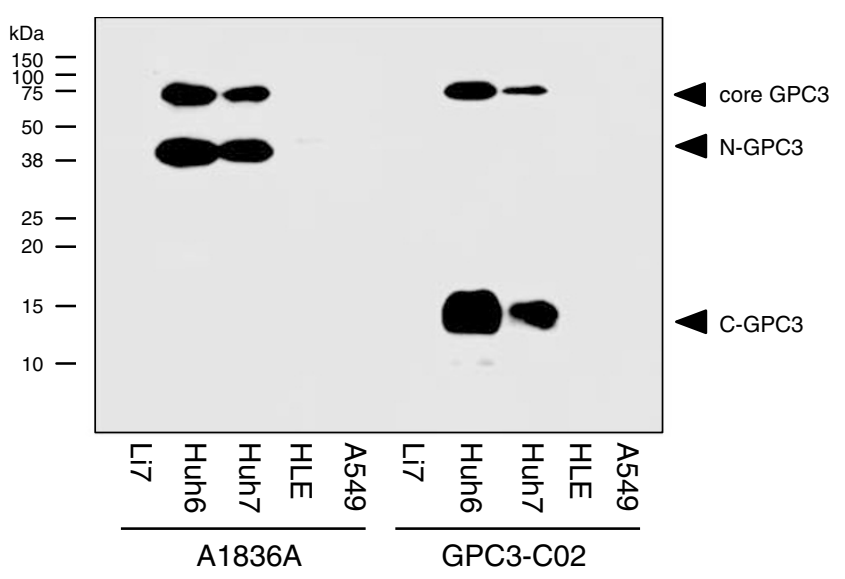

Figure 1 Immunoblotting of glypican 3 in cancer cell lines. The anti-glypican 3 (GPC3) antibody A1836A detects a $69 \mathrm{kDa}$ of core GPC3 and $40 \mathrm{kDa}$ of a cleaved N-terminal fragment of GPC3 in human cancer cell lines of hepatocyte lineage, Huh6 and Huh7, but not in Li7 or HLE. GPC3-C02, an antibody that recognizes the C-terminal region of GPC3, demonstrates two bands corresponding to the core GPC3 and $15 \mathrm{kDa}$ protein in Huh6 and Huh7. Proteins of 69,40 , and $15 \mathrm{kDa}$ are not detectable in a lung cancer cell line, A549.

(Figure 1). Northern blotting detected mRNA of GPC3 in Huh6 and Huh7, but not in Li7 and HLE. ${ }^{20}$

A1836A (the anti-GPC3 antibody that recognizes the N-terminal region of GPC3) detected $69 \mathrm{kDa}$ of core GPC3 and $40 \mathrm{kDa}$ of a cleaved N-terminal fragment of GPC3 in Huh6 and Huh7. GPC3-C02 (the anti-GPC3 antibody that recognizes the Cterminal region of GPC3) detected two bands corresponding to core GPC3 and a $15 \mathrm{kDa}$ protein in Huh6 and Huh7. We further confirmed that the 69, 40 and $15 \mathrm{kDa}$ proteins were not detectable in a lung cancer cell line, A549 and another 20 cancer cell lines that did not express GPC3 (data not shown).

\section{Immunohistochemistry of GPC3}

When GPC3-C02 and A1836A antibodies were used to assess GPC3 expression in formalin-fixed, paraffin-embedded tissue sections, both antibodies displayed similar immunoreactivity. Table 1 summarizes the immunohistochemical results of GPC3 expression in normal, non-neoplastic, and neoplastic liver tissues.

\section{GPC3 Expression in Fetal and Adult Liver Tissues}

The expression of GPC3 was identified in the fetal liver (Figure 2a) from the 18 to the 39 gestational week. Immunostaining was most prominent adjacent to canaliculi. The pattern and intensity of the staining remained unchanged throughout the fetal life. On the other hand, no GPC3 expression was observed in any of the adult liver tissues. 
Table 1 Glypican-3 expression in normal, non-neoplastic, and neoplastic liver tissues

\begin{tabular}{|c|c|c|c|}
\hline & \multirow[t]{2}{*}{$\begin{array}{c}\text { No. of } \\
\text { specimens }\end{array}$} & \multicolumn{2}{|c|}{$\begin{array}{c}\text { No. of } \\
\text { positive cases }\end{array}$} \\
\hline & & + & ++ \\
\hline Adult normal liver & 25 & 0 & 0 \\
\hline Fetal liver & 9 & 0 & 9 \\
\hline Fulminant hepatitis & 9 & 0 & 0 \\
\hline (regenerative nodule) & 1 & 1 & 0 \\
\hline Autoimmune hepatitis & 2 & 0 & 0 \\
\hline Primary sclerosing cholangitis & 1 & 0 & 0 \\
\hline Chronic hepatitis (viral) & 13 & 0 & 0 \\
\hline Primary biliary cirrhosis & 2 & 0 & 0 \\
\hline Cirrhosis (viral) & 43 & 0 & 0 \\
\hline Focal nodular hyperplasia & 3 & 1 & 0 \\
\hline Adenoma & 7 & 0 & 0 \\
\hline Dysplastic nodule (low grade) & 8 & 2 & 0 \\
\hline Dysplastic nodule (high grade) & 8 & 6 & 0 \\
\hline Hepatocellular carcinoma & 56 & 0 & 47 \\
\hline Well/moderately/poorly & $18 / 29 / 9$ & $0 / 0 / 0$ & $14 / 24 / 9$ \\
\hline$<3.0 \mathrm{~cm} /=$ or $>3.0 \mathrm{~cm}$ & $29 / 27$ & $0 / 0$ & $27 / 20$ \\
\hline Cholangiocellular carcinoma & 16 & 0 & 0 \\
\hline Carcinoid tumor & 1 & 0 & 0 \\
\hline Hepatoblastoma & 6 & 3 & 3 \\
\hline Metastatic colon carcinoma & 23 & 1 & 0 \\
\hline
\end{tabular}

All cases with $\geq 10 \%$ positive cells were regarded as either focally positive $(+)(10-50 \%)$ or diffusely positive $(++)(>50 \%)$.

\section{GPC3 Expression in Non-Neoplastic Liver Lesions}

GPC3 expression was undetectable in all but one of the non-neoplastic tissue samples with diffuse inflammatory changes. In one of the nine cases of fulminant hepatitis, the protein was expressed in a tiny focus of regenerative nodule consisiting of small non-neoplastic hepatocytes (Figure 2b-d). GPC3 immunostaing was similarly prominent adjacent to canaliculi (Figure 2d), as in the fetal liver. Autoimmune hepatitis, primary sclerosing cholangitis, chronic viral hepatitis, primary biliary cirrhosis, and viral cirrhosis were all completely negative for GPC3.

One out of three cases with focal nodular hyperplasia exhibited weak and focal staining in some of the hyperplastic hepatocytes.

\section{GPC3 Expression in Neoplastic Liver Lesions}

\section{Hepatocyte lineage}

Weak and focal staining was detected in two of eight low-grade (Figure 3a, b) and six of eight high-grade dysplastic nodules. No GPC3 was detected in any of the seven liver cell adenomas. GPC3 expression was specifically observed in malignant hepatocytes, however, and hepatocellular carcinoma showed diffusely positive staining in 47 of 56 cases (84\%) (Figure 3c, d). GPC3 immunoreactivity was intense both in the cellular membranes and cytoplasms of the hepatocellular carcinomas, but no reactivity was observed in other tissues integrated within or adjacent to the tumors (non-neoplastic hepatocytes, bile ducts, and blood vessels). GPC3 was expressed in 14 of 18 well-differentiated (78\%), 24 of 29 moderately differentiated ( $83 \%)$, and in nine of nine poorly differentiated HCCs $(100 \%)$. As for the staining pattern of GPC3, canalicular pattern as seen in fetal liver tissues was observed in two and four cases of well and moderately differentiated carcinomas, respectively, suggesting that the canalicular pattern did not parallel with morphological differentiation. The size of the hepatocellular carcinoma was not significantly related to the GPC3 expression $(P=0.0733)$. GPC3 was completely negative in sarcomatoid hepatocellular carcinomas. One case showed typical well-differentiated trabecular type hepatocellular carcinoma in addition to sarcomatoid hepatocellular carcinoma. It was also interesting to note that GPC3 was positive in the typical welldifferentiated hepatocellular carcinoma component and negative in the sarcomatoid hepatocellular carcinoma component in the same slide.

Examination of the hepatoblastomas revealed GPC3 positivity (Figure 3e) in the epithelial components in all of the specimens examined, whether the epithelial components were fetal or embryonal. Stainig pattern was not restricted to canalicular pattern, which was observed in the fetal liver. As for the mesenchymal components, there was no immunostaing identified.

\section{Other cell lineages}

In contrast to hepatocellular carcinoma, carcinoid tumor and cholangiocellular carcinomas were negative for GPC3. GPC3 was detected in only one of the 23 metastatic lesions of colorectal carcinoma, in the form of focal staining of cytoplasmic pattern (Figure 3f). The primary colon carcinoma of this case was histologically the same, and there was also focal cytoplasmic immunoreactivity for GPC3 in the primary site.

\section{Hep and AFP Immunostaining of Hepatocellular Carcinoma}

Table 2 summarizes the immunohistochemical results of GPC3, Hep, and AFP expression in 45 cases of hepatocellular carcinoma. Hep-immunostaining was observed diffusely in the hepatocytes of both hepatocellular carcinoma and non-neoplastic liver. On the other hand, AFP-immunostaining was specific to hepatocellular carcinoma, but its frequency was significantly lower than that of GPC3 in well and moderately differentiated carcinomas ( $P=0.0048$ and $P=0.0025$, respectively). 

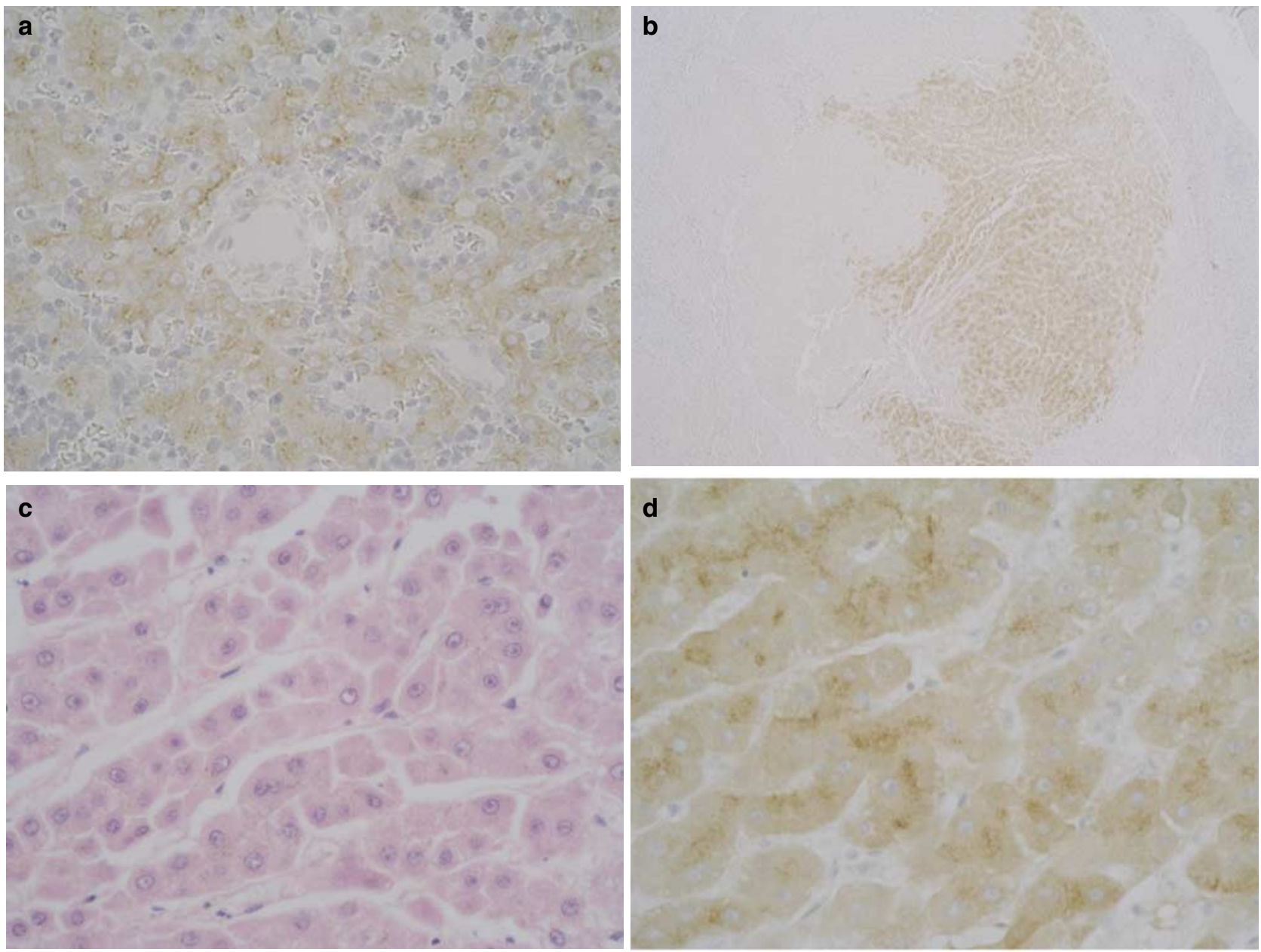

Figure 2 Immunohistochemistry of glypican 3 in non-neoplastic liver tissues. (a) Glypican 3 (GPC3) immunohistochemical staining of fetal liver. Fetal liver tissue shows preferential canalicular staining with an anti-GPC3 monoclonal antibody. (GPC3 immunohistochemical staining $(\times 400)$ ). $(\mathbf{b}-\mathbf{d})$ GPC3 immunohistochemistry of fulminant hepatitis. A tiny regenerative nodule (b) consisting of small hepatocytes(c), positive for GPC3 (b, d) with canalicular pattern, was observed in a case of fulminant hepatitis. Partial discohesiveness of hepatocytes was due to a saline artefact $(\mathbf{b}(\times 40))(\mathbf{c}, \mathbf{d}(\times 400))(\mathbf{b}, \mathbf{d}$, GPC3 immunohistochemical staining; c, HE staining).

\section{Discussion}

GPC3 is a member of the glypican family of glycosylphosphatidyl inositol-anchored cell-surface heparan sulfate proteoglycans. ${ }^{6}$ The messenger RNA (mRNA) levels of GPC3 have recently been found to be markedly elevated in a large proportion of hepatocellular carcinomas, and this elevation is apparently independent of AFP. ${ }^{13,16,18}$ In our immunoblotting and immunostaining of the paraffin-embedded tissue sections, the two monoclonal antibodies against GPC3 (GPC3-C02 and A1836A) specifically recognized GPC3 molecule in the fetal liver and malignant hepatocytes both in vitro and in vivo. As no GPC3 was detected in the hepatocytes of chronic hepatitis, liver cirrhosis, or normal adult liver, we could reliably characterize GPC3 as an oncofetal protein, at least in the liver. In our preliminary studies with the two monoclonal antibodies in adult tissues other than the liver, using an autopsy tissue array, GPC3 expression was not observed in systemic organs except for weak expression in a group of bronchiolar epithelial cells of the lung.

We also found GPC3 expression in an area of marked regenerative activity in the setting of fulminant hepatitis. The staining pattern was comparable with that in the fetal liver, and such a pattern was only occasional in hepatocellular carcinomas. Further investigation may reveal the relationship of this regenerative phenomenon to the molecular biology of hepatocellular carcinoma. Although GPC3 was originally identified as a suppressor of cell proliferation, ${ }^{21,22}$ it also acts as a negative regulator of inhibitory growth factors such as BMP-7. ${ }^{20,23}$ Intracellular distribution of GPC3 might be related to such an altered function. Thus, the GPC3 expression in malignant hepatocytes appears to indicate not only the reactivation of a fetal phenotype, but possibly some other biologically significant event in the carcinogenesis of the liver, as well. 

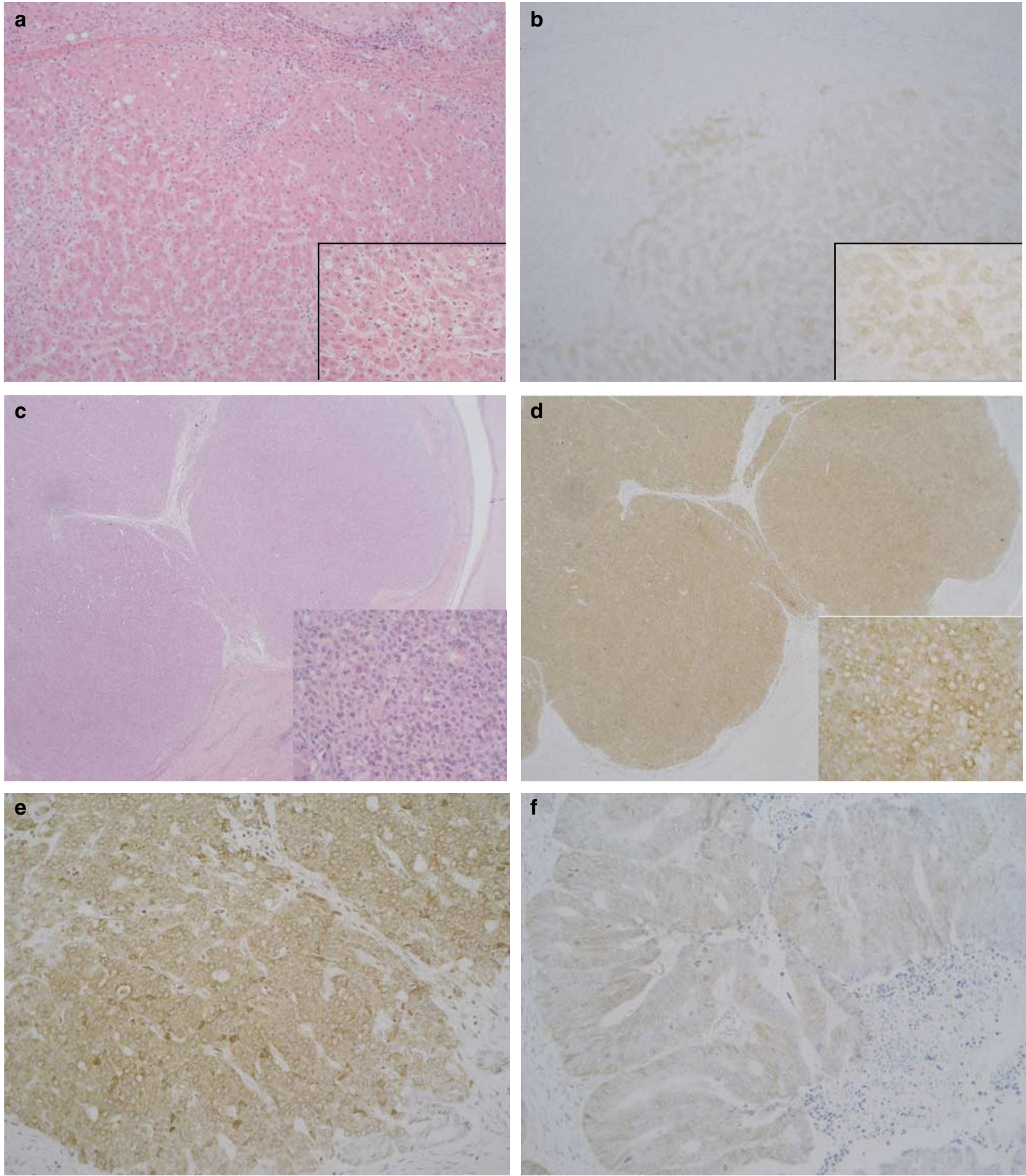

Figure 3 Glypican 3 in neoplastic liver tissues. (a and b: GPC3 immunohistochemistry of hepatic dysplastic nodule (low grade). A nodule showing dysplastic change (a) is focally and weakly stained with an anti-GPC3 monoclonal antibody (b). (a, HE staining $(\times 200$, inset: $\times 400)$; b, GPC3 immunohistochemical staining $(\times 200$, inset: $\times 400)$ ). (c and d: GPC3 immunoreactivity of hepatocellular carcinoma. A nodular lesion of the case was found in a case with hepatic cirrhosis (c). A diffuse strong staining is observed in the hepatocellular carcinoma, but not in cirrhotic nodules (d). Insets: higher magnification of hepatocellular carcinoma showing cellular details. (c, HE staining $(\times 20$, inset: $\times 400)$; d, GPC3 immunohistochemical staining $(\times 20$, inset: $\times 400)$ ). (e) GPC3 immunohistochemistry of hepatoblastoma. Epithelial component in hepatoblastoma shows diffuse cytoplasmic staining for GPC3 (GPC3 immunohistochemical staining $(\times 200)$ ). (f) GPC3 immunohistochemistry of hepatic metastasis of colorectal carcinoma. Focal staining is rarely observed in an adenocarcinoma with tubular structure (GPC3 immunohistochemical staining $(\times 200)$ ). 
Table 2 Expression of glypican-3, hepatocyte antigen, and alphafetoprotein expression in hepatocellular carcinoma

\begin{tabular}{lccc}
\hline & Glypican-3 & $\begin{array}{c}\text { Hepatocyte } \\
\text { antigen }\end{array}$ & $\begin{array}{c}\text { Alpha- } \\
\text { fetoprotein }\end{array}$ \\
\hline $\begin{array}{l}\text { Hepatocellular } \\
\text { carcinoma }\end{array}$ & $39 / 45$ & $38 / 45$ & $17 / 45$ \\
$\quad$ Well & $10 / 13$ & $13 / 13$ & $2 / 13^{*}$ \\
$\begin{array}{l}\text { Moderately } \\
\text { Poorly }\end{array}$ & $23 / 26$ & $21 / 26$ & $12 / 26^{* *}$ \\
Non-neoplastic liver & $6 / 6$ & $4 / 6$ & $3 / 6$ \\
\hline
\end{tabular}

${ }^{*} P=0.0048$ and ${ }^{*} P=0.0025$, when compared with the corresponding figure of glypican-3.

GPC3 expression in well-differentiated hepatocellular carcinoma showed several features in the present study. It was observed in $78 \%$ of all the well-differentiated carcinomas examined, and it exhibited a diffuse and uniform distribution within individual tumors. The expression appeared to bear no relation to the size of the hepatocellular carcinoma and was observed even in small-sized tumors. On the other hand, the expression was only focal and weak in focal nodular hyperplasia and low- and high-grade dysplastic nodules. No GPC3 was detected in the liver cell adenomas. All of these findings recommend the GPC3 immunohistochemistry as a marker to differentiate hepatocellular carcinoma from benign hepatocyte nodules, especially in the cases of core needle biopsy where only small amounts of tissue can be taken. CD34, an intercellular adhesion protein, has also been recently used for this purpose. ${ }^{3,4,24}$ The endothelial phenotype in hepatocellular carcinoma along the sinusoidal capillarization exhibits a loss of fenestrate and deposition of basement membrane, as well as alterations of cell-cell and cell-matrix interaction. It thus appears that the GPC3 expression in the hepatocytes might be induced by the same mechanism that induces the CD34 expression in the endothelial cells, namely, the adaptation to the capillarization of hepatocellular carcinoma. From a diagnostic perspective, CD34 immunohistochemistry is reportedly useful to differentiate hepatocellular carcinoma from low-grade dysplastic nodule, and our results demonstrate that GPC3-immunohistochemistry differentiates hepatocellular carcinoma from high-grade dysplastic nodule, as well. The use of both immunohistochemical analyses may therefore prove complementary in diagnosing low-grade dysplastic nodule, high-grade dysplastic nodule, and hepatocellular carcinoma. In liver cell adenoma, although there is significant CD34 reactivity, ${ }^{4}$ there is no reactivity for GPC3. Therefore, GPC3 is very useful to differentiate hepatocellular carcinoma from liver cell adenoma.

At the other end of the spectrum in the pathological diagnosis of hepatocellular carcinoma, moderately and poorly differentiated hepatocellular carcinomas may be difficult to differentiate from cholangiocellular carcinoma and metastatic carcinoma. GPC3 was strikingly specific for hepatocellular carcinoma in the present study, with no GPC3 observed in cholangiocellular carcinoma or bile duct structures of fetal and adult livers with or without regenerative change. Hepatocyte antigen (Hep), alpha-fetoprotein (AFP), cytokeratin protein profiling, and the presence of intercellular canaliculi demonstrated by the monoclonal antibodies carcinoembryonic antigen Gold 5 and B-lymphocyte marker CD10 have all been used as hepatocyte markers. In this study, we revealed that GPC3 was more sensitive than AFP and more specific than Hep-immunohistochemistry. Therefore, the antibodies directed against GPC3 have great promise as an immunohistochemical marker of hepatocellular carcinoma in routine histological examination and as targets in monoclonal antibody-based hepatocellular carcinoma therapy. With the wide range of options of markers, we can benefit further by determining whether the hepatocyte panel including GPC3 demonstrates the various stages of hepatocyte differentiation, and if it does, by defining the significance of such a differentiation in tumor behavior and the prognosis of hepatocellular carcinoma.

One case in the present study exhibited focal positivity for GPC3 in the liver metastasis of a colorectal carcinoma. Evaluation of the corresponding primary carcinoma demonstrated that the colon carcinoma of this exceptional case also showed focal immunoreactivity for GPC3. The staining pattern was cytoplasmic in both cases, suggesting that staining pattern of GPC3, membranous or cytoplasmic, is potentially useful for the differentiation between primary and metastatic carcinomas. However, we should be cautious that both patterns could be observed when much more amount of GPC3 is expressed in the carcinoma cells. GPC3 might be expressed in a manner similar to AFP, that is, as an oncofetal protein in carcinomas of various organs, especially those of the gastro-intestinal tract. Thus, it is also interesting to extend our study of hepatocyte differentiation in carcinomas of the extrahepatic organs using GPC3 with other hepatocyte markers, as hepatoid carcinoma has been morphologically recognized in a variety of organs.

In conclusion, the monoclonal antibodies GPC3C02 and A1836A specifically recognized GPC3 in fetal and malignant hepatocytes in Western blot and immunohistochemical analyses. GPC3 was confirmed to be one of the oncofetal proteins now attracting attention for their promise both as markers of hepatocellular carcinoma in routine histological examination and as targets in monoclonal antibodybased hepatocellular carcinoma therapy.

\section{Acknowledgements}

We are grateful to Ms M Saito and Ms Y Ohno for their technical assistance. This work was supported 
by a Grants-in Aid for Scientific Research (B)12557051 and Scientific Research on Priority Areas(C)13218019 from the Ministry of Education, Culture, Sports, Science and Technology, and Health and Labour Sciences Research Grants on Hepatitis. This study was carried out as a part of The Technology Development for Analysis of Protein Expression and Interaction in Bioconsortia on R\&D of New Industrial Science and Technology Frontiers that was overseen by the industrial Science, Technology and Environmental Policy Bureau, Ministry of Economy, Trade \& Industry, and delegated to New Energy Development Organization.

\section{References}

1 Schafer DF, Sorrell MF. Hepatocellular carcinoma. Lancet 1999;353:1253-1257.

2 Okuda K. Hepatocellular carcinoma. J Hepatol 2000; 32:225-237.

3 Park YN, Yang CP, Fernandez GJ, et al. Neoangiogenesis and sinusoidal 'capillarization' in dysplastic nodules of the liver. Am J Surg Pathol 1998;22:656-662.

4 Kong CS, Appenzeller M, Ferrell LD. Utility of CD34 reactivity in evaluating focal nodular hepatocellular lesions sampled by fine needle aspiration biopsy. Acta Cytol 2000;44:218-222.

5 Chu PG, Ishizawa S, Wu E, et al. Hepatocyte antigen as a marker of hepatocellular carcinoma: an immunohistochemical comparison to carcinoembryonic antigen, CD10, and alpha-fetoprotein. Am J Surg Pathol 2002; 26:978-988.

6 Filmus J, Selleck SB. Glypicans:proteoglycans with a surprise. J Clin Invest 2001;108:497-501.

7 Grisaru S, Cano-Gauci DF, Tee J, et al. Glypican-3 modulates BMP- and FGF-mediated effects during renal branching morphogenesis. Dev Biol 2001;231:31-46.

8 Paine-Saunders S, Viviano BL, Zupicich J, et al. Glypican-3 controls cellular responses to Bmp4 in limb patterning and skeletal development. Dev Biol 2000;225:179-187.

9 Zhang Z, Coomans C, David G. Membrane heparan sulfate proteoglycan-supported FGF2-FGFR1 signaling. Evidence in support of the 'cooperative end structures’ model. J Biol Chem 2001;276:41921-41929.

10 Lin $\mathrm{H}$, Huber R, Schlessinger $\mathrm{D}$, et al. Frequent silencing of the GPC3 gene in ovarian cancer cell lines. Cancer Res 1999;59:807-810.
11 Murthy SS, Shen T, De Rienzo A, et al. Expression of GPC3, an X-linked recessive overgrowth gene, is silenced in malignant mesothelioma. Oncogene 2000; 19:410-416.

12 Xiang YY, Ladeda V, Filmus J. Glypican -3 expression is silenced in human breast cancer. Oncogene 2001; 20:7408-7412.

13 Hsu HC, Cheng W, Lai PL. Cloning and expression of a developmentally regulated transcript MXR7 in hepatocellular carcinoma: biological significance and temporospatial distribution. Cancer Res 1997;57: 5179-5184.

14 Lage H, Dietel M, Froschle G, et al. Expression of the novel mitoxantrone resistance associated gene MXR7 in colorectal malignancies. Int J Clin Pharmacol Ther 1998;36:58-60.

15 Saikali Z, Sinnett D. Expression of glypican3(GPC3) in embryonal tumors. Int J Cancer 2000;89:418-422.

16 Zhu ZW, Friess H, Wang L, et al. Enhanced glypican-3 expression differentiates the majority of hepatocellular carcinomas from benign hepatic disorders. Gut 2001; 48:558-564.

17 International Working Party. Terminology of nodular hepatocellular lesions. Hepatology 1995;22:983-993.

18 Midorikawa Y, Ishikawa S, Iwanari H, et al. Glypican3 , overexpressed in hepatocellular carcinoma, modulates FGF2 and BMP-7 signaling. Int J Cancer 2003;103: 455-465.

19 Hippo Y, Watanabe K, Watanabe A, et al. Identification of soluble amino terminal fragment of glypican-3 as a serological marker for early stage hepatocellular carcinoma. Cancer Res 2004;64:2418-2423.

20 Watanabe A, Hippo Y, Taniguchi H, et al. An opposing view on WWOX protein function as a tumor suppressor. Cancer Res 2003;63:8629-8633.

21 Gonzalez AD, Kaya M, Shi W, et al. OCI-5/GPC3, a glypican encoded by a gene that is mutated in the Simpson-Golabi-Behmel overgrowth syndrome, induces apoptosis in a cell line-specific manner. J Cell Biol 1998;141:1407-1414.

22 Song HH, Shi W, Filmus J. OCI-5/rat glypican-3 binds to fibroblast growth factor-2 but not to insulinlike growth factor-2. J Biol Chem 1997;272:75747577.

23 Piscione TD, Phan T, Rosenblum ND. BMP7 controls collecting tubule cell proliferation and apoptosis via Smad1-dependent and -independent pathways. Am J Physiol Renal Physiol 2001;280:F19-F33.

24 Stahl J, Voyvodic F. Biopsy diagnosis of malignant versus benign liver 'nodules': new helpful markers. An update. Adv Anat Pathol 2000;7:230-239. 